\title{
Risk Contours Map For Risk Bounded Motion Planning Under Perception Uncertainties
}

\author{
Ashkan M. Jasour, Brian C. Williams \\ MIT, Computer Science and Artificial Intelligence Laboratory \\ \{jasour,williams@csail.mit.edu\}
}

\begin{abstract}
In this paper, we introduce "risk contours map" that contains the risk information of different regions in uncertain environments. Risk is defined as the probability of collision of robots with obstacles in presence of probabilistic uncertainties in location, size, and geometry of obstacles. We use risk contours to obtain safe paths for robots with guaranteed bounded risk. We formulate the problem of obtaining risk contours as a chance constrained optimization. We leverage the theory of moments and nonnegative polynomials to provide a convex optimization in the form of sum of squares optimization. Provided approach deals with nonconvex obstacles and probabilistic bounded and unbounded uncertainties. We demonstrate the performance of the provided approach by solving risk bounded motion planning problems.
\end{abstract}

\section{INTRODUCTION}

Concern for safety is one of the dominant issues that arises when planning in presence of uncertainties and disturbances. To ensure safety, one needs to leverage robust and risk aware approaches to deal with uncertainties. In this paper, we address risk bounded motion planning in presence of uncertainties in perception of robots where we have probabilistic uncertainties in location, size, and geometry of obstacles. To ensure safety, instead of working with an ordinary map that shows obstacles and free regions in the environment, we suggest to build a map, called "risk contours", that shows the risk information of different regions of the uncertain environment. Risk is defined as the probability of collision of robots with obstacles in presence of probabilistic uncertainties. Such maps can be used to design trajectories for robots to avoid risky regions and, in general, to plan risk bounded motions. In risk bounded motion planning, one aims at designing trajectories whose probability of failure (risk) is bounded by predefined value (admissible risk level), ([1], [2], [3], [4]).

The problem of risk computation in continuous space is numerically challenging because it requires multivariate integrals with respect to the probability distributions of uncertainties over nonconvex sets that describe the obstacle regions. Such integrals, in general, do not have any closed form solutions. Given the obstacles and

This work was supported by Boeing grant MIT-BA-GTA-1. probability distribution of uncertainties, one can use sampling based methods and also Boole's inequality to estimate the risk. Sampling based methods like Monte Carlo techniques ([5], [6], [7]) do not provide any analytical bounds on the risk. Hence, these approaches can not be directly used in risk bounded motion planning where we need strict upper bounds of the risk [4]. Also, Boole's inequality provides conservative upper bounds of the risk in presence of linear convex obstacles. In ([1], [2], [3]) Boole's inequality is used for risk bounded motion planning in presence of Gaussian distributions and convex obstacles. In [8], risk-aware cost is defined based on Gaussian distributions and logistic function to characterize the safe distance between the vehicles. In [4], we propose risk estimation method in presence of nonconvex regions where polynomial optimizations are used to provide upper and lower bounds on probability of violation of nonconvex safety constraints.

In this paper, we introduce "risk contours map" to model uncertain environments. To the best of our knowledge, this problem has not been addressed before. Risk contours map shows the risk information of different regions in uncertain environments. To build such map, we define the notion of " $\Delta$-risk contour" that represents a set of points in the environment whose risk is less than or equal to the predefined risk level $\Delta$. Risk contours map is a collection of such " $\Delta$-risk contours" with different risk levels $\Delta$. Note that, we can not directly use risk estimation techniques like [4] because we need to look for risk contours in the environment and, at the same time, their associated risk.

In this paper, we provide a systematic way to construct risk contours in presence of nonconvex obstacles and both bounded and unbounded probabilistic uncertainties. We provide convex optimizations in the form of "sum of squares" (SOS) optimizations. In SOS optimization, we look for polynomials whose coefficients satisfy linear matrix inequalities. SOS optimization has different applications in control and motion planning of autonomous systems and robots, (e.g., see [4], [9], [10], [11], [12], [13], [14]). To obtain SOS optimizations, we leverage our recent results on "chance constrained optimization". 
In ([15], [16]), building on the theory of moments and nonnegative polynomials, we provide convex optimizations to solve chance constrained optimizations. Using these results, we obtain outer and inner approximations of " $\Delta$-risk contours".

The outline of the paper is as follows: in Section II, we cover the notation adopted in the paper and present preliminary results on moments and SOS optimization; In section III, we formulate the problem of risk contours construction and present an illustrative example; Section IV shows the application of risk contours map in risk bounded motion planning; Sections V and VI detail the proposed technique to find outer and inner approximations of risk contours and provide illustrative examples; In Section VII, we present numerical results, followed by some concluding remarks given in Section VIII.

\section{NOTATION AND PRELIMINARY RESUltS}

This section covers notation and some basic definitions used in this paper ([17], [18], [19], [15]).

Polynomials: Let $\mathbb{R}[x]$ be the set of real polynomials in the variables $x \in \mathbb{R}^{n}$. Given $\mathcal{P} \in \mathbb{R}[x]$, we represent $\mathcal{P}$ as $\sum_{\alpha \in \mathbb{N}^{n}} p_{\alpha} x^{\alpha}$ using the standard basis $\left\{x^{\alpha}\right\}_{\alpha \in \mathbb{N}^{n}}$ of $\mathbb{R}[x]$, and $\mathbf{p}=\left\{p_{\alpha}\right\}_{\alpha \in \mathbb{N}^{n}}$ denotes the polynomial coefficients and $\alpha \in \mathbb{N}^{n}$, e.g., $\alpha=\left(\alpha_{1}, \ldots, \alpha_{n}\right)$ with $\alpha_{i}$ in $\mathbb{N}$. Also, let $\mathbb{R}_{\mathrm{d}}[x] \subset \mathbb{R}[x]$ denotes the set of polynomials of degree at most $d \in \mathbb{N}$. For example a polynomial of degree at most $d=2$ in $x_{1}$ and $x_{2}(n=2)$ can be represented as $\mathcal{P}=\sum_{\alpha=\left(\alpha_{1}, \alpha_{2}\right)} p_{\alpha_{1} \alpha_{2}} x_{1}^{\alpha_{1}} x_{2}^{\alpha_{2}} \in \mathbb{R}_{2}[x]$ where $\alpha=\left(\alpha_{1}, \alpha_{2}\right) \in\{[0,0],[1,0],[0,1],[2,0],[1,1],[0,2]\}$ and $p_{\alpha_{1} \alpha_{2}}$ are coefficients. In general, any given $\mathcal{P} \in$ $\mathbb{R}_{\mathrm{d}}[x]$ has $S_{n, d}:=\left(\begin{array}{c}d+n \\ n\end{array}\right)$ number of coefficients. In this paper, we use polynomials to represent obstacles and safe regions [4]. For example the set $\left\{\left(x_{1}, x_{2}\right): 1-x_{1}^{2}-x_{2}^{2} \geq\right.$ $0\}$ represents a circular-shaped obstacle.

Moments: Given a probability distribution $\mu(x)$ in $\mathbb{R}^{n}$ and $\alpha \in \mathbb{N}^{n}$, moment of order $\alpha$ of $\mu(x)$ is defined as $y_{\alpha_{1}, \alpha_{2}, \ldots, \alpha_{n}}=E\left[x_{1}^{\alpha_{1}} x_{2}^{\alpha_{2}} \ldots x_{n}^{\alpha_{n}}\right]=\int x_{1}^{\alpha_{1}} x_{2}^{\alpha_{2}} \ldots x_{n}^{\alpha_{n}} \mu(x) d x$, ([17], [18], [15]). For example, first and second moments of a probability distribution in $\mathbb{R}$, i.e., $y_{1}=E[x]$ and $y_{2}=E\left[x^{2}\right]$, represent the mean and variance of the distribution. As another example, given $n=2$ and $\alpha=2, \mathbf{y}=\left[y_{00}, y_{10}, y_{01}, y_{20}, y_{11}, y_{02}\right]$ represents the moment sequence up to order 2 of a distribution in $\mathbb{R}^{2}$.

Sum of Squares Optimization: Polynomial $\mathcal{P}(x)$ : $\mathbb{R}^{n} \rightarrow \mathbb{R}$ is a sum of squares (SOS) polynomial if it can be written as a sum of finitely many squared polynomials, i.e., $\mathcal{P}(x)=\sum_{j=1}^{m} h_{j}(x)^{2}$ for some $m<\infty$ and $h_{j}(x) \in$ $\mathbb{R}[x]$ for $1 \leq j \leq m$, ([17], [18], [19]). SOS condition is a convex constraint that can be represented as a linear matrix inequality in terms of coefficients of polynomial, i.e., $\mathcal{P}(x) \in S O S \rightarrow \mathcal{P}(x)=\mathbf{x}^{\prime} A \mathbf{x}$, where $\mathbf{x}$ is the vector of standard basis and $A$ is a positive semidefinite matrix in terms of the coefficients of the polynomial, ([19], [18], [17]).

Optimization over nonnegative polynomials involves a linear cost function in terms of coefficients of polynomials and polynomial nonnegativity constraints, i.e., $\min _{\mathbf{p}} \mathbf{c}^{\prime} \mathbf{p}$, s.t. $\mathcal{P}(x) \geq 0$ on $\mathcal{K}=\left\{x: g_{j}(x) \geq\right.$ $0, j=1, \ldots, \ell\}$, where $\mathbf{p}$ is coefficients vector of polynomial $\mathcal{P}$ and $\mathcal{K}$ is a compact set described by given polynomials $g_{j}(x), j=1, \ldots, \ell$. Nonnegativity constraint "P $(x) \geq 0$ on $\forall x \in \mathcal{K}$ " can be relaxed to a convex SOS constraint " $\mathcal{P}(x)=h_{0}(x)^{2}+\sum_{j=1}^{\ell} h_{j}(x)^{2} g_{j}(x)$ " (sufficient condition), ([19], [18], [17]). In this paper, we provide SOS optimizations to construct risk contours.

\section{RISK CONTOURS}

Let $\chi_{o b s}$ be an uncertain obstacle defined in terms of polynomial $\mathcal{P}$ as follows [4]:

$$
\chi_{o b s}(\omega):=\{x \in \chi: \mathcal{P}(x, \omega) \leq 0\}
$$

where, $\chi \subset \mathbb{R}^{n}, \omega \in \Omega \subset \mathbb{R}^{m}$ are uncertain parameters with known probability distribution $\mu_{\omega}(\omega)$ and $\mathcal{P}: \mathbb{R}^{n} \times$ $\mathbb{R}^{m} \rightarrow \mathbb{R}$ is a given polynomial in $x \in \chi$ and $\omega \in \Omega$. In general, $\chi_{o b s}(\omega)$ represents an nonconvex obstacle with probabilistic uncertain parameters $\omega$, e.g., obstacle with uncertain size, location, or geometry.

We can represent uncertain safe region as follows:

$$
\chi_{\text {safe }}(\omega)=\chi-\chi_{\text {obs }}(\omega)
$$

where, "-" is the set difference. We define the risk as the probability of collision with uncertain obstacle. Let $\Delta \in$ $[0,1]$ be the admissible risk level. Then, we define the " $\Delta$-risk contour" for uncertain obstacle $\chi_{o b s}(\omega)$ denoted by $\mathcal{C}_{r}^{\Delta}$ as the set of all points in the environment whose probability of collision with the uncertain obstacle is less than or equal to $\Delta$. More precisely,

$$
\mathcal{C}_{r}^{\Delta}:=\left\{x \in \chi: \operatorname{Prob}_{\mu_{\omega}(\omega)}\left(x \in \chi_{o b s}(\omega)\right) \leq \Delta\right\}
$$

Computation of such set is hard, because any point $x^{*}$ in the set $\mathcal{C}_{r}^{\Delta}$ should satisfy the "chance constraint" $\operatorname{Prob}_{\mu_{\omega}}\left(x^{*} \in \chi_{\text {obs }}(\omega)\right)=\int_{\left\{\omega \in \Omega: \mathcal{P}\left(x^{*}, \omega\right) \leq 0\right\}} \mu_{\omega}(\omega) d \omega \leq \Delta$. Such constraint involves a multivariate integral over a nonconvex set which is computationally challenging. In this paper, we provide a systematic numerical procedure to compute outer and inner approximations of $\Delta$-risk contours for given uncertain obstacle and probability distributions of uncertainties.

Remark 1: Although unsafe obstacles are defined by just one polynomial, the approach proposed in this paper can be extended to more complex sets involving multiple polynomials. This assumption is only done to simplify the exposition. 
Assumption 1: Provided approach deals with both unbounded and bounded probabilistic uncertainties. In the case of unbounded uncertainties, we assume that probability distributions are moment determinate, where distributions can be completely determined by their moments [20], e.g, normal distribution can completely be determined by its first and second moments.

\section{A. Illustrative Example}

Consider the following simple illustrative example where $\chi=[-1,1]^{2}$ and $\Omega=[-1,1]$. The set $\chi_{\text {obs }}(\omega)=\left\{x \in \mathbb{R}^{2}: x_{1}^{2}+x_{2}^{2}-\omega^{2} \leq 0\right\}$ represents an circular-shaped obstacle with uncertain radius $\omega$ with uniform probability distribution over $[0.3,0.4]$, i.e., $\mu_{\omega}=$ $U[0.3,0.4]$. Then, the set $\left\{x \in \chi: x_{1}^{2}+x_{2}^{2}-0.4^{2} \geq 0\right\}$ represents $\mathcal{C}_{r}^{\Delta=0}$, a set of points whose probability of collision with $\chi_{o b s}(\omega)$ is 0 , because $\operatorname{Prob}(\omega \geq 0.4)=0$. Also, the set $\left\{x \in \chi: x_{1}^{2}+x_{2}^{2}-0.35^{2} \geq 0\right\}$ represents $\mathcal{C}_{r}^{\Delta=0.5}$, a set of points whose probability of collision is less than or equal to 0.5 , because $\operatorname{Prob}(\omega \geq 0.35)=0.5$.

\section{Risk Bounded Motion Planning Using Risk CONTOURS MAP}

In risk bounded motion planning, we are looking for a trajectory $P(t)$ between given initial point $x_{0} \in \chi$ and goal point $x_{T} \in \chi$ whose probability of collision with each obstacle is bounded by given admissible risk level $\Delta,([1],[2],[3])$. More precisely, trajectory $P(t)$ should satisfy the following constraints:

i) Boundary Conditions: $P(0)=x_{0}, P(T)=x_{T}$

ii) Chance Constraints: $\operatorname{Prob}\left\{P(t) \in \chi_{o b s_{i}}\left(\omega_{i}\right)\right\} \leq$ $\Delta, i=1, \ldots, o, \forall t \in[0, T]$, where $\chi_{o b s_{i}}\left(\omega_{i}\right), i=1, \ldots, o$ are uncertain obstacles of the form (1).

To satisfy the chance constraints, we can use risk contours of obstacles $\mathcal{C}_{r_{i}}^{\Delta}, i=1, \ldots, o$. Hence, the chance constraints can be replaced by deterministic constraints as follows:

$$
P(t) \in\left\{x \in \chi: \cap_{i=1}^{o} \mathcal{C}_{r_{i}}^{\Delta}\right\}, \forall t \in[0, T]
$$

Now, we can use any path planning methods like $R R T^{*}$ and $P R M$ [21] to find a path satisfying boundary conditions and deterministic constraint (4).

\section{CONVEX Optimization FOR OUter APPROXIMATION OF RISK CONTOURS}

In this section, we provide a systematic numerical procedure to compute an outer approximation of the $\Delta$ risk contour denoted by $\hat{\mathcal{C}}_{r}^{\Delta}$ for a given uncertain obstacle $\chi_{o b s}(\omega)$ and probability distribution of uncertainties $\mu_{\omega}(\omega)$. Given the uncertain obstacle and uncertain safe set, the following holds true for the $\Delta$-risk contour:

$$
\begin{aligned}
\mathcal{C}_{r}^{\Delta} & =\left\{x \in \chi: \operatorname{Prob}_{\mu_{\omega}(\omega)}\left(x \in \chi_{o b s}(\omega)\right) \leq \Delta\right\} \\
& =\left\{x \in \chi: \operatorname{Prob}_{\mu_{\omega}(\omega)}\left(x \in \chi_{\text {safe }}(\omega)\right) \geq 1-\Delta\right\}
\end{aligned}
$$

The main idea is to replace the chance constraint of the $\Delta$-risk contour in (5) with a deterministic constraint. For this, we will replace the "Prob $\mu_{\omega}\left(x \in \chi_{\text {safe }}(\omega)\right) \geq 1-\Delta$ " with " $\mathcal{P}_{\text {outer }}(x) \geq 1-\Delta$ " and represent the outer approximation set as $\hat{\mathcal{C}}_{r}^{\bar{\Delta}}=\left\{x \in \chi: \mathcal{P}_{\text {outer }}(x) \geq 1-\Delta\right\}$, where $\mathcal{P}_{\text {outer }}: \mathbb{R}^{n} \rightarrow \mathbb{R}$ is a polynomial. To construct the polynomial $\mathcal{P}_{\text {outer }}(x)$, we leverage our recent results on "chance constrained optimization" based on the theory of moments and SOS polynomials ([15], [16]).

For this purpose, we define the following sets:

$$
\begin{aligned}
\mathcal{K}_{o b s}= & \{(x \in \chi, \omega \in \Omega): \mathcal{P}(x, \omega) \leq 0\} \\
& \mathcal{K}_{\text {safe }}=\chi \times \Omega-\mathcal{K}_{\text {obs }}
\end{aligned}
$$

Note that $\mathcal{K}_{o b s} \subset \chi \times \Omega$ and $\chi_{o b s} \subset \chi$. Consider the following SOS optimization.

$$
\begin{aligned}
\min _{\hat{\mathcal{P}} \in \mathbb{R}_{d}[x, \omega]} & \int_{\chi \times \Omega} \hat{\mathcal{P}}(x, \omega) \mu_{\omega}(\omega) d \omega d x, \\
\text { s.t. } & \hat{\mathcal{P}}(x, \omega)-1 \geq 0 \text { on } \quad\left\{\forall(x, \omega) \in \mathcal{K}_{\text {safe }}\right\} \\
& \hat{\mathcal{P}}(x, \omega) \geq 0 .
\end{aligned}
$$

where $\hat{\mathcal{P}}(x, \omega) \in \mathbb{R}_{d}[x, \omega]$ is a unknown polynomial of order $d$ in $x$ and $\omega$. Optimization (8) has a linear cost function in terms of moments up to order $d$ and polynomial nonnegativity constraints. Hence, it can be relaxed to a SOS optimization.

Now, we construct the polynomial $\mathcal{P}_{\text {outer }}(x)$ and the set $\hat{\mathcal{C}}_{r}^{\Delta}$ in terms of $d$-order polynomial solution of SOS optimization (8) as follows:

$$
\begin{gathered}
\mathcal{P}_{\text {outer }}(x)=\int_{\Omega} \hat{\mathcal{P}}(x, \omega) \mu_{\omega}(\omega) d \omega \\
\hat{\mathcal{C}}_{r}^{\Delta}=\left\{x \in \chi: \mathcal{P}_{\text {outer }}(x) \geq 1-\Delta\right\}
\end{gathered}
$$

The following Theorem holds true.

Theorem 1: The set $\hat{\mathcal{C}}_{r}^{\Delta}$ in (10) is an outer approximation of $\Delta$-risk contour $\mathcal{C}_{r}^{\Delta}$ in (3) and converges asymptotically as $d$ increases.

Proof: Appendix A.

We can interpret the obtained results in (8) and (9) as follows. For any fixed point $x^{*} \in \chi$, polynomial $\hat{\mathcal{P}}(x, \omega)$ in (8) is an upper bound approximation of the indicator function of the set $\left\{\left(x^{*}, \omega\right) \in \mathcal{K}_{\text {safe }}\right\}$ denoted by $\mathcal{I}_{\left\{\left(x^{*}, \omega\right) \in \mathcal{K}_{\text {safe }}\right\}}$, i.e., $\mathcal{I}_{\left\{\left(x^{*}, \omega\right) \in \mathcal{K}_{\text {safe }}\right\}}=1$ for $\left(x^{*}, \omega\right) \in$ $\mathcal{K}_{\text {safe }}$ and 0 otherwise. Then, $\mathcal{P}_{\text {outer }}\left(x^{*}\right)$ is an upper bound on the probability that $x^{*} \in \chi_{\text {safe }}$, i.e., $\operatorname{Prob}_{\mu_{\omega}}\left(x^{*} \in \chi_{\text {safe }}(\omega)\right)=\operatorname{Prob}_{\mu_{\omega}}\left(\left(x^{*}, \omega\right) \in \mathcal{K}_{\text {safe }}\right)=$ $\int_{\left\{\left(x^{*}, \omega\right) \in \mathcal{K}_{\text {safe }}\right\}} \mu_{\omega}(\omega) d \omega=\int_{\Omega} \mathcal{I}_{\left\{\left(x^{*}, \omega\right) \in \mathcal{K}_{\text {safe }}\right\}} \mu_{\omega}(\omega) d \omega \leq$ $\int_{\Omega} \hat{\mathcal{P}}\left(x^{*}, \omega\right) \mu_{\omega}(\omega) d \omega=\mathcal{P}_{\text {outer }}\left(x^{*}\right)$. Hence, the set $\{x \in$ $\left.\chi: \mathcal{P}_{\text {outer }}(x) \geq 1-\Delta\right\}$ describes the outer approximation of the set of points whose probability of being 


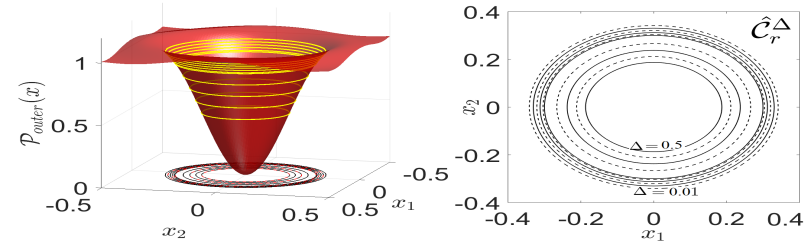

Fig. 1: Polynomial $\mathcal{P}_{\text {outer }}(x)$ obtained by solving SOS optimization (8) and Eq. (9). Outside of the circles represents outer approximations of $\Delta$-risk contours, i.e., $\hat{\mathcal{C}}_{r}^{\Delta}=\left\{x \in \chi: \mathcal{P}_{\text {outer }}(x) \geq 1-\Delta\right\}$
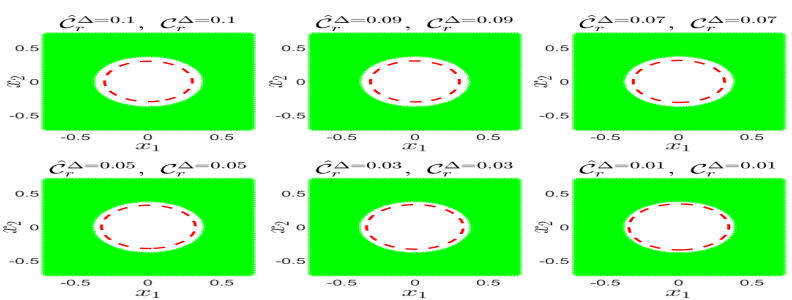

Fig. 2: True $\Delta$-risk contour $\mathcal{C}_{r}^{\Delta}$ (green) and outer approximation $\hat{\mathcal{C}}_{r}^{\Delta}=\left\{x \in \chi: \mathcal{P}_{\text {outer }}(x) \geq 1-\Delta\right\}$ (outside of the dashed-line)

in the uncertain safe region is greater than or equal to $1-\Delta$, (for more information on indicator function based probability estimation see [4]).

Note that SOS optimization in (8) is independent of the risk level $\Delta$. Hence, we can use its solution to construct the outer approximation of the risk contours for different risk levels $\Delta$ as in (10).

\section{A. Illustrative Example}

Consider unsafe obstacle of example in III-A. To obtain outer approximations of $\Delta$-risk contours, i.e., $\hat{\mathcal{C}}_{r}^{\Delta}$, we solve the optimization in (8) with $d=20$ using Yalmip, which is a MATLAB-based toolbox aimed at SOS optimization [22], with Mosek solver. The computation time to solve the SOS optimization is approximately $150(\mathrm{sec})$. Then, according to (9), $\mathcal{P}_{\text {outer }}(x)=$ $\int_{-1}^{1} \hat{\mathcal{P}}(x, \omega) \mu_{\omega}(\omega) d \omega=\int_{0.3}^{0.4} \hat{\mathcal{P}}(x, \omega) 10 d \omega$. According to (10), outer approximation of $\Delta$-risk contour set is obtained in terms of polynomial $\mathcal{P}_{\text {outer }}(x)$. Figure 1 shows the obtained $\mathcal{P}_{\text {outer }}(x)$ and obtained $\hat{\mathcal{C}}_{r}^{\Delta}$, for the risk levels $\Delta=[0.5,0.4,0.3,0.2,0.1,0.09,0.07,0.05,0.03,0.01]$. To verify the obtained result, we use Monte Carlo simulation. The details of the Monte Carlo simulation are discussed in section VII. Figure 2 shows $\Delta$-risk contours obtained by Monte Carlo simulation and also obtained outer approximations $\hat{\mathcal{C}}_{r}^{\Delta}$, for the risk levels $\Delta=[0.1,0.09,0.07,0.05,0.03,0.01]$. Note that based on Theorem 1, tighter sets can be obtained by increasing the polynomial order $d$ in optimization (8).

\section{CONVEX Optimization FOR INNER APPROXIMATION OF RISK CONTOURS}

Consider uncertain obstacle (1) and uncertain safe set (2). Then, the following holds true: $\chi=\left\{x \in \chi: \operatorname{Prob}\left(x \in \chi_{\text {obs }}(\omega)\right) \leq \Delta\right\}+$ $\left\{x \in \chi: \operatorname{Prob}\left(x \in \chi_{o b s}(\omega)\right)>\Delta\right\}$. Hence, $\quad \mathcal{C}_{r}^{\Delta}=$ $\chi-\left\{x \in \chi: \operatorname{Prob}\left(x \in \chi_{o b s}(\omega)\right)>\Delta\right\}$. So, an outer approximation of the set $\left\{x \in \chi: \operatorname{Prob}\left(x \in \chi_{o b s}(\omega)\right)>\Delta\right\}$ results in an inner approximation of $\mathcal{C}_{r}^{\Delta}$.

In the previous section, we used SOS optimization in (8) and (10) to obtain outer approximations of the set $\left\{x \in \chi: \operatorname{Prob}\left(x \in \chi_{\text {safe }}(\omega)\right) \geq 1-\Delta\right\}$. We can apply the same methodology to obtain outer approximations of the set $\left\{x \in \chi: \operatorname{Prob}\left(x \in \chi_{o b s}(\omega)\right)>\Delta\right\}$. For this, we define the following SOS optimization.

$$
\begin{aligned}
& \min _{\overline{\mathcal{P}} \in \mathbb{R}_{d}[x, \omega]} \int_{\chi \times \Omega} \overline{\mathcal{P}}(x, \omega) \mu_{\omega}(\omega) d \omega d x, \\
& \text { s.t. } \overline{\mathcal{P}}(x, \omega)-1 \geq 0 \text { on } \quad\left\{\forall(x, \omega) \in \mathcal{K}_{o b s}\right\} \\
& \overline{\mathcal{P}}(x, \omega) \geq 0 .
\end{aligned}
$$

where $\overline{\mathcal{P}}(x, \omega) \in \mathbb{R}_{d}[x, \omega]$ is a unknown polynomial of order $d$ in $x$ and $\omega$ and $\mathcal{K}_{o b s}$ is the set defined in (6). Now, we construct the polynomial $\mathcal{P}_{\text {inner }}(x)$ and an inner approximation of $\Delta$-risk contour denoted by $\overline{\mathcal{C}}_{r}^{\Delta}$ as follows:

$$
\begin{gathered}
\mathcal{P}_{\text {inner }}(x)=\int_{\Omega} \overline{\mathcal{P}}(x, \omega) \mu_{\omega}(\omega) d \omega \\
\overline{\mathcal{C}}_{r}^{\Delta}=\left\{x \in \chi: \mathcal{P}_{\text {inner }}(x) \leq \Delta\right\}
\end{gathered}
$$

The following Theorem holds true.

Theorem 2: The set $\overline{\mathcal{C}}_{r}^{\Delta}$ in (13) is an inner approximation of $\Delta$-risk contour $\mathcal{C}_{r}^{\Delta}$ in (3) and converges asymptotically as $d$ increases.

Proof: Appendix B.

Similar to $\mathcal{P}_{\text {outer }}(x)$ in the previous section, for any $x^{*} \in \chi$, polynomial, $\mathcal{P}_{\text {inner }}\left(x^{*}\right)$ is an upper bound on the probability that $x^{*} \in \chi_{o b s}$, i.e., $\operatorname{Prob}_{\mu_{\omega}}\left(x^{*} \in\right.$ $\left.\chi_{\text {obs }}(\omega)\right) \leq \mathcal{P}_{\text {inner }}\left(x^{*}\right)$ and $\left\{x \in \chi: \mathcal{P}_{\text {inner }}(x)>\Delta\right\}$ is an outer approximation of the set of points whose probability of collision is greater than $\Delta$. Hence, the set $\left\{x \in \chi: \mathcal{P}_{\text {inner }}(x) \leq \Delta\right\}$ describes an inner approximation of $\Delta$-risk contour.

\section{A. Illustrative Example}

Consider unsafe obstacle of provided example in III-A. To obtain an inner approximation of $\Delta$-risk contours, i.e., $\overline{\mathcal{C}}_{r}^{\Delta}$, we solve SOS optimization in (11) with $d=20$. Then, according to (12), $\mathcal{P}_{\text {inner }}(x)=$ $\int_{-1}^{1} \overline{\mathcal{P}}(x, \omega) \mu_{\omega}(\omega) d \omega=\int_{0.3}^{0.4} \overline{\mathcal{P}}(x, \omega) 10 d \omega$. According to (13), inner approximation of $\Delta$-risk contour is obtained in terms of polynomial $\mathcal{P}_{\text {inner }}(x)$. Figure 3 shows the obtained $\mathcal{P}_{\text {inner }}(x)$ and obtained $\overline{\mathcal{C}}_{r}^{\Delta}$ for the risk levels 


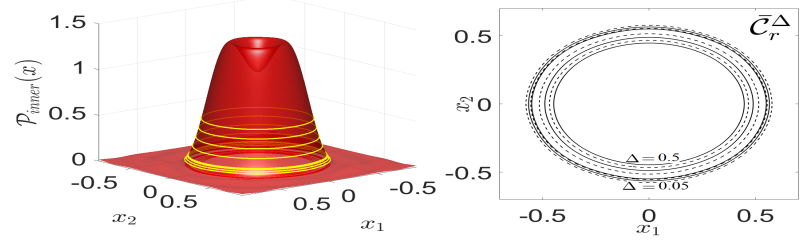

Fig. 3: Polynomial $\mathcal{P}_{\text {inner }}(x)$ obtained by solving SOS optimization (11) and Eq. (12). Outside of the circles represents inner approximations of $\Delta$-risk contours, i.e., $\overline{\mathcal{C}}_{r}^{\Delta}=\left\{x \in \chi: \mathcal{P}_{\text {inner }}(x) \leq \Delta\right\}$.
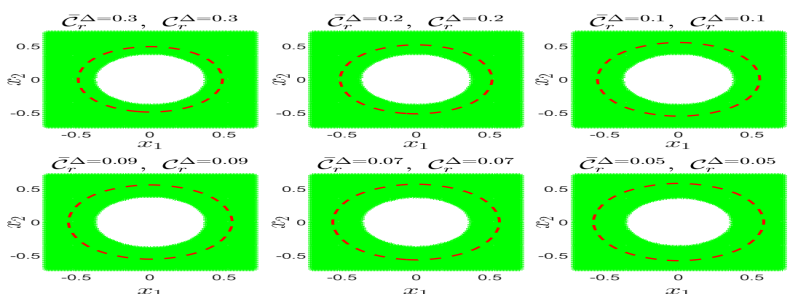

Fig. 4: True $\Delta$-risk contour $\mathcal{C}_{r}^{\Delta}$ (green) and inner approximation $\overline{\mathcal{C}}_{r}^{\Delta}=\left\{x \in \chi: \mathcal{P}_{\text {inner }}(x) \leq \Delta\right\}$ (outside of the dashed-line)

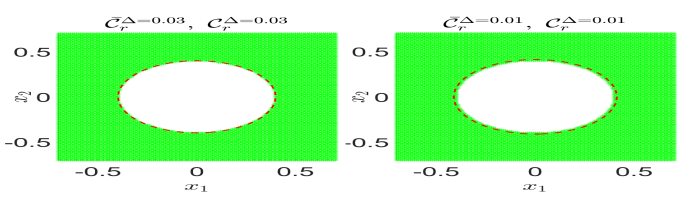

Fig. 5: True $\Delta$-risk contours (green) and inner approximations described by scaled $\Delta$-risk contours as $\overline{\mathcal{C}}_{r}^{\Delta}=0.01=\hat{\mathcal{C}}_{r}^{\Delta}=0.01\left(0.83 x_{1}, 0.83 x_{2}\right)$ and $\overline{\mathcal{C}}_{r}^{\Delta}=0.03=$ $\hat{\mathcal{C}}_{r}^{\Delta}=0.03\left(0.83 x_{1}, 0.83 x_{2}\right)$.

$\Delta=[0.5,0.4,0.3,0.2,0.1,0.09,0.07,0.05]$. Figure 4 shows $\Delta$-risk contours obtained by Monte Carlo simulation and also obtained inner approximations $\overline{\mathcal{C}}_{r}^{\Delta}$ for the risk levels $\Delta=[0.3,0.2,0.1,0.09,0.07,0.05]$.

Remark 2: Note that based on Theorem 2 tighter sets can be obtained by increasing the polynomial order $d$ in optimization (11). An alternative approach to obtain a tighter set for small $d$ is to use "scaled $\Delta$-risk contours" that is obtained by rescaling the inner and outer approximation sets by introducing a scaling factor $s_{x}$, i.e., $\hat{\mathcal{C}}_{r}^{\Delta}\left(s_{x} \times x\right)=\left\{x \in \chi: \mathcal{P}_{\text {outer }}\left(s_{x} \times x\right) \geq 1-\Delta\right\}$ and $\overline{\mathcal{C}}_{r}^{\Delta}\left(s_{x} \times x\right)=\left\{x \in \chi: \mathcal{P}_{\text {inner }}\left(s_{x} \times x\right) \leq \Delta\right\}$. For example, Figure 5 shows tight inner approximations of $\Delta$-risk contours for $\Delta=[0.03,0.01]$ described by scaled outer approximation sets, i.e., $\overline{\mathcal{C}}_{r}^{\Delta}=0.01=\hat{\mathcal{C}}_{r}^{\Delta=0.01}\left(0.83 x_{1}, 0.83 x_{2}\right)$ and $\overline{\mathcal{C}}_{r}^{\Delta}=0.03=\hat{\mathcal{C}}_{r}^{\Delta}=0.03\left(0.83 x_{1}, 0.83 x_{2}\right)$. Monte Carlo simulation is used to verify the obtained scaled $\Delta$-risk contour.

\section{IMPLEMENTATION AND NUMERICAL RESULTS}

In this section, three examples are presented that illustrate the performance of the proposed approach. Given the mathematical description of uncertain obstacles in (1) and probability distributions of uncertainties, we use Yalmip [22] and Mosek to solve the provided SOS optimization and construct an inner approximation of $\Delta$-risk contours. The computations in this section were performed on a laptop with Intel i $2.7 \mathrm{GHz}$ processors and 8 GB RAM. As described in IV, by obtaining $\Delta$-risk contours for different risk levels $\Delta$ in the offline step, one can use any path planning methods to find feasible risk bounded paths for robots. In this paper, we assume that polynomial representation of obstacles are given. One can use SOS based approaches in ([9], [23]), to construct such descriptions of obstacles from point cloud data obtained by sensors. As an alternative approach, one can model the obstacles with simple ellipsoids. Also, we assume that probability distributions of uncertainties are obtained by developed probabilistic models or intention recognition algorithms (e.g., [24]).

To test the accuracy of the obtained results, we use Monte Carlo simulation [15] to estimate $\mathcal{C}_{r}^{\Delta}$. For this purpose, we first uniformly grid $\chi$ into $\bar{N}$ gridpoints ( $\bar{N}$ depending on the desired precision). Let $\left\{x^{(i)}\right\}_{i=1}^{\bar{N}} \subset \chi$ denote the points in the uniform grid. Next, for each grid point $x^{(i)}$, we sample from the probability distributions of uncertain parameters $\mu_{\omega}$ supported on $\Omega$. Let $\left\{\omega^{(i, k)}\right\}_{k=1}^{N_{i}}$ be $N_{i}$ i.i.d. sample of random parameter $\omega$. Then, we approximate $\mathbf{P}_{\text {risk }}=$ $\operatorname{Prob}_{\mu_{\omega}}\left(x^{(i)} \in \chi_{o b s}(\omega)\right)=\int_{\left\{\omega \in \Omega: \mathcal{P}\left(x^{(i)}, \omega\right) \leq 0\right\}} \mu_{\omega} d \omega$ by $P_{N_{i}}^{(i)}:=\frac{1}{N_{i}} \sum_{k=1}^{N_{i}} \mathbf{I}\left(x^{(i)}, \omega^{(i, k)}\right)$, where $\mathbf{I}(x, \omega)=$ 1 if $(x, \omega) \in\{(x, \omega): \mathcal{P}(x, \omega) \leq 0\}$, and $\mathbf{I}(x, \omega)=$ 0 otherwise. For each $x^{(i)}$, we chose sample size $N_{i}$ such that $P_{N_{i}}^{(i)}$ becomes stagnant to further increase in $N_{i}$. Then, the set of sampled points $x^{(i)}$ whose estimated risk satisfy $\mathbf{P}_{\text {risk }} \leq \Delta$ represents $\mathcal{C}_{r}^{\Delta}$.

\section{A. Example 1}

An uncertain unsafe region is modeled as $\chi_{o b s}(\omega)=$ $\left\{\left(x_{1}, x_{2}\right) \in \chi:-39.0625 x_{1}^{4}+3.125 x_{1}^{2}-2.25 x_{2}^{2}+\right.$ $0.01+0.5 \omega \leq 0\}$ with uncertain parameter $\omega$ that has a Beta probability distribution over $[0,1]$ with parameters $\alpha=1.1$ and $\beta=5$, i.e., $\mu_{\omega}=\operatorname{Beta}(1.1,5)$. Figure 6-a shows the cluttered environment and $\chi_{o b s}(\omega)$ for different values of uncertain parameter $\omega=0$ and 1 .

To obtain inner approximations of $\Delta$-risk contours, i.e., $\overline{\mathcal{C}}_{r}^{\Delta}$, we first solve the SOS optimization in (11) with $d=24$ to find polynomial $\mathcal{P}_{\text {inner }}(x)$. The computation time to solve the SOS optimization is approximately $500(\mathrm{sec})$. Figure 7-a shows the obtained $\mathcal{P}_{\text {inner }}(x)$. Then, we use $\mathcal{P}_{\text {inner }}(x)$ to construct the $\overline{\mathcal{C}}_{r}^{\Delta}$ according to (12) and (13) for different value of risk levels $\Delta$. Figure 7-b shows the obtained inner 


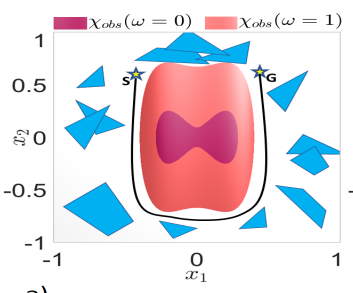

a)

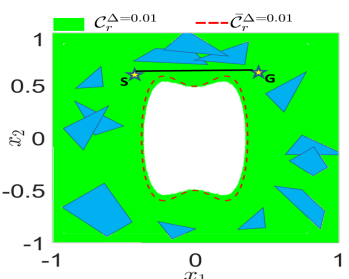

b)

Fig. 6: a) Cluttered environment and $\chi_{o b s}(\omega)$ for $\omega=0$ and 1 . The constructed path between the start and goal points is obstacle free for all possible values of $\omega$ (robust motion planning). b) True $\Delta=0.01$ risk contour (green) and inner approximation $\overline{\mathcal{C}}_{r}^{\Delta}=0.01$ (outside of the dashed-line). The constructed path between the start and goal points is risk bounded.
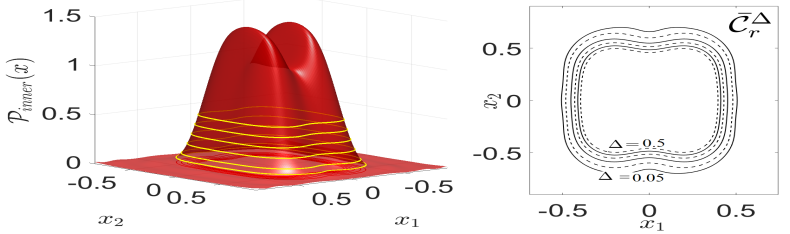

Fig. 7: a) Polynomial $\mathcal{P}_{\text {inner }}(x)$ obtained by solving SOS optimization (11) and Eq. (12). b) Outside of the contours represent inner approximation of $\Delta$-risk contours, i.e., $\overline{\mathcal{C}}_{r}^{\Delta}=$ $\left\{x \in \chi: \mathcal{P}_{\text {inner }}(x) \leq \Delta\right\}$.

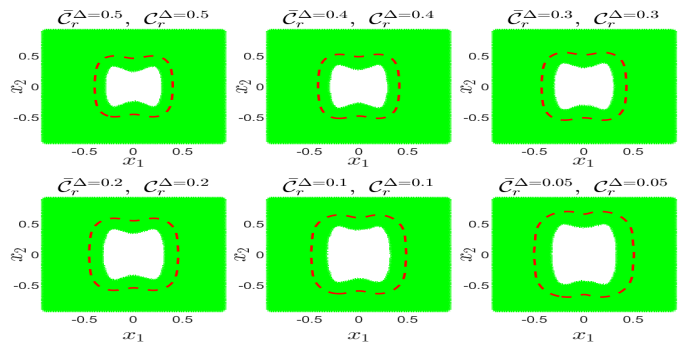

Fig. 8: True $\Delta$-risk contours $\mathcal{C}_{r}^{\Delta}$ (green) and inner approximations $\overline{\mathcal{C}}_{r}^{\Delta}=\left\{x \in \chi: \mathcal{P}_{\text {inner }}(x) \leq \Delta\right\}$ (outside of the dashed-line).

approximation of $\Delta$-risk contours for the risk levels of $\Delta=[0.5,0.4,0.3,0.2,0.1,0.05]$. Also Figure 8 compares the obtained inner approximations with actual $\Delta$ risk contours obtained by Monte Carlo simulation.

Moreover, Figure 6-b shows the obtained tight inner approximation using the scaled $\Delta$-risk contour described in Remark 2, for risk level $\Delta=0.01$ as $\overline{\mathcal{C}}_{r}^{\Delta=0.01}=$ $\hat{\mathcal{C}}_{r}^{\Delta=0.01}\left(0.89 x_{1}, 0.69 x_{2}\right)$. We use the obtained $\overline{\mathcal{C}}_{r}^{\Delta=0.01}$ to construct a path between start and goal points with bounded risk of 0.01 shown in Figure 6-b. We compare the obtained result using risk bounded motion planning (Figure 6-b) with robust motion planning (Figure 6-a) where the designed path should be obstacle free for all possible values of the uncertain parameter $\omega$.

\section{B. Example 2}

In the presence of multiple uncertain obstacles, one needs to obtain the the risk contours for each obstacles and use the obtained sets to construct the risk bounded path as in (4). In such scenarios, it is easier to work with uncertain safe region instead of multiple uncertain obstacles. For example, in the following example, shown in Figure 9, we have several dynamic obstacles whose locations are uncertain. The safe region between the obstacles is modeled as $\chi_{\text {safe }}(\omega)=\left\{\left(x_{1}, x_{2}\right) \in \chi\right.$ : $\left.-\left(x_{1}^{4}+\left(x_{2}-0.4\right)^{4}+\left(x_{2}-0.4\right)^{3}-0.1(\omega-0.5)\right) \geq 0\right\}$ with uncertain parameter $\omega$ that has a Triangular probability distribution over $[0,1]$ with the peak point 0.5 , i.e., $\mu_{\omega}=\operatorname{Tri}(0.5)$, (for deterministic and convex version of this problem see [25]).

To obtain outer approximations of the $\Delta$-risk contours, we first solve the SOS optimization in (8) with $d=24$ to find polynomial $\mathcal{P}_{\text {outer }}(x)$, (shown in Figure 10a). The computation time to solve the SOS optimization is approximately $500(\mathrm{sec})$. Then, we construct the $\hat{\mathcal{C}}_{r}^{\Delta}$ according to (9) and (10) for different value of risk levels $\Delta$. Figure 10-b shows the obtained outer approximation of $\Delta$-risk contours for the risk levels of $\Delta=[0.9,0.8,0.7,0.6,0.5,0.4,0.3,0.2,0.1,0.05,0.01]$. Also Figure 11 compares the obtained inner approximations with actual $\Delta$-risk contours obtained by Monte Carlo simulation.

To obtain inner approximations of the $\Delta$-risk contours, we first solve the SOS optimization in (11) with $d=24$ to find polynomial $\mathcal{P}_{\text {inner }}(x)$, (shown in Figure 12-a). Then, we construct the $\overline{\mathcal{C}}_{r}^{\Delta}$ according to (12) and (13) for different value of risk levels $\Delta$. Figure 12-b shows the obtained inner approximation of $\Delta$-risk contours for risk levels of $\Delta=[0.9,0.8,0.7,0.6,0.5,0.4,0.3,0.2,0.1]$. Also, Figure 13 compares the obtained inner approximations with actual $\Delta$-risk contours obtained by Monte Carlo simulation. Moreover, Figure 14 shows the obtained tight inner approximation using the scaled $\Delta$-risk contour described in Remark 2, for risk levels $\Delta=[0.1,0.05,0.01]$ as $\overline{\mathcal{C}}_{r}^{\Delta=0.1}=$ $\overline{\mathcal{C}}_{r}^{\Delta=0.45}\left(0.8 x_{1}, 0.9 x_{2}\right), \overline{\mathcal{C}}_{r}^{\Delta=0.05}=\overline{\mathcal{C}}_{r}^{\Delta=0.35}\left(0.75 x_{1}, 0.85 x_{2}\right)$, $\overline{\mathcal{C}}_{r}^{\Delta=0.01}=\overline{\mathcal{C}}_{r}^{\Delta=0.3}\left(0.75 x_{1}, 0.85 x_{2}\right)$.

\section{Example 3}

Due to the wind disturbances, location of a flying drone (moving obstacle) for time steps $k=1,2,3$ are modeled as $\chi_{o b s}\left(\omega_{1_{k}}, \omega_{2_{k}}, \omega_{3_{k}}\right)=$ $\left\{x \in \mathbb{R}^{3}:\left(x_{1}-\omega_{1_{k}}\right)^{2}+\left(x_{2}-\omega_{2_{k}}\right)^{2}+\left(x_{3}-\omega_{3_{k}}\right)^{2}-0.2^{2} \leq 0\right\}$, where the uncertain parameters have the following Normal distributions: $\mu_{\omega_{1}}=$ $\mathcal{N}(-0.3,0.02), \mu_{\omega_{1}}=\mathcal{N}(0,0.06), \mu_{\omega_{1}}=\mathcal{N}(0.3,0.1)$, 


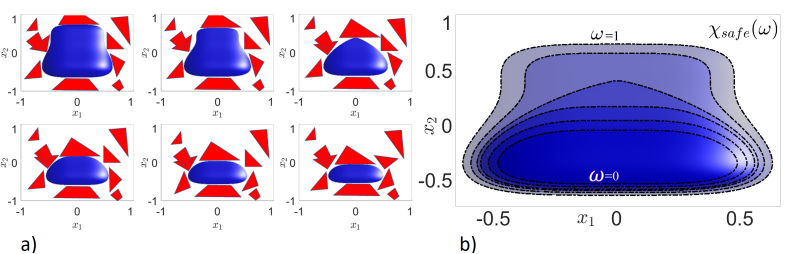

Fig. 9: a) Uncertain safe region (blue) between dynamic obstacles (red) with uncertain locations. b) Uncertain safe region for different valuse of uncertain parameter $\omega$.
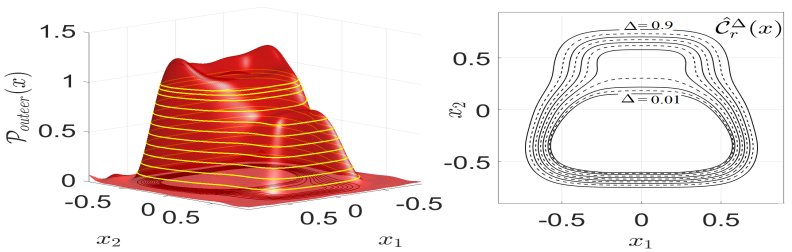

Fig. 10: a) Polynomial $\mathcal{P}_{\text {outer }}(x)$ obtained by solving SOS optimization (8) and Eq. (9). b) Inside of the contours represent outer approximation of $\Delta$-risk contours, i.e., $\hat{\mathcal{C}}_{r}^{\Delta}=$ $\left\{x \in \chi: \mathcal{P}_{\text {outer }}(x) \geq 1-\Delta\right\}$.

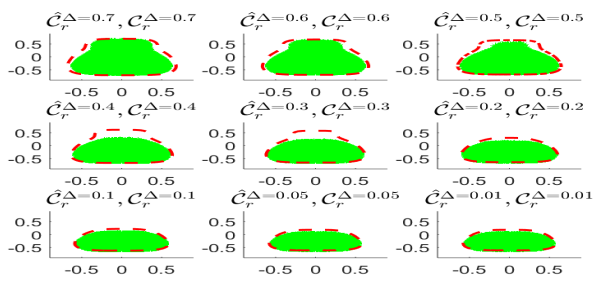

Fig. 11: True $\Delta$-risk contour set $\mathcal{C}_{r}^{\Delta}$ (green) and outer approximation $\hat{\mathcal{C}}_{r}^{\Delta}=\left\{x \in \chi: \mathcal{P}_{\text {outer }}(x) \geq 1-\Delta\right\}$ (inside of the dashed-line).
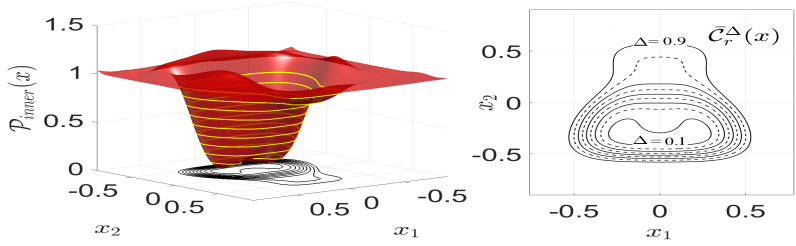

Fig. 12: a) Polynomial $\mathcal{P}_{\text {inner }}(x)$ obtained by solving SOS optimization (11) and Eq. (12). b) Inside of the contours represent inner approximation of $\Delta$-risk contours, i.e., $\overline{\mathcal{C}}_{r}^{\Delta}=\left\{x \in \chi: \mathcal{P}_{\text {inner }}(x) \leq \Delta\right\}$.

$\mu_{\omega_{21}}=\mathcal{N}(-0.3,0.02), \mu_{\omega_{2}}=\mathcal{N}(0,0.06), \mu_{\omega_{2}}=\mathcal{N}(0.3,0.1)$, $\mu_{\omega_{3_{1}}}=\mathcal{N}(-0.3,0.02), \mu_{\omega_{3}}=\mathcal{N}(0,0.02), \mu_{\omega_{3}}=\mathcal{N}(0.3,0.02)$.

We obtain inner approximations of the $\Delta=0.1$-risk contour, set of points whose probability of collision with the drone is less than or equal to 0.1 , for time steps $k=1,2,3$. At each time steps, we solve SOS optimization according to the probability distributions of the uncertain parameters. Figure 15 shows the obtained results.

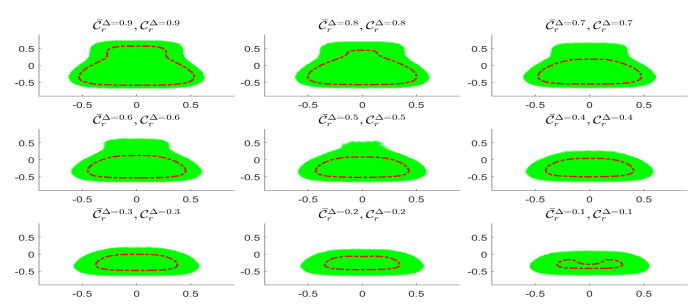

Fig. 13: True $\Delta$-risk contour $\mathcal{C}_{r}^{\Delta}$ (green) and inner approximation $\overline{\mathcal{C}}_{r}^{\Delta}=\left\{x \in \chi: \mathcal{P}_{\text {inner }}(x) \leq \Delta\right\}$ (inside of the dashed-line).
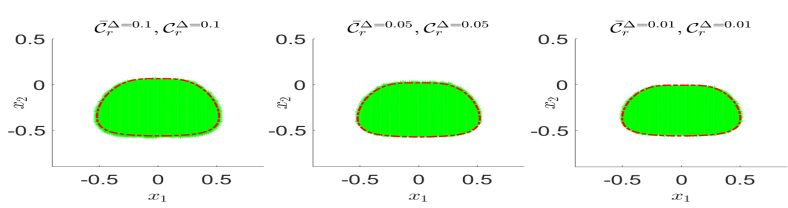

Fig. 14: Inner approximations of $\mathcal{C}_{r}^{\Delta}$ described by scaled $\Delta$ risk contours, $\overline{\mathcal{C}}_{r}^{\Delta}=0.1=\overline{\mathcal{C}}_{r}^{\Delta}=0.45\left(0.8 x_{1}, 0.9 x_{2}\right), \overline{\mathcal{C}}_{r}^{\Delta}=0.05=$ $\overline{\mathcal{C}}_{r}^{\Delta}=0.35\left(0.75 x_{1}, 0.85 x_{2}\right), \overline{\mathcal{C}}_{r}^{\Delta}=0.01=\overline{\mathcal{C}}_{r}^{\Delta}=0.3\left(0.75 x_{1}, 0.85 x_{2}\right)$.

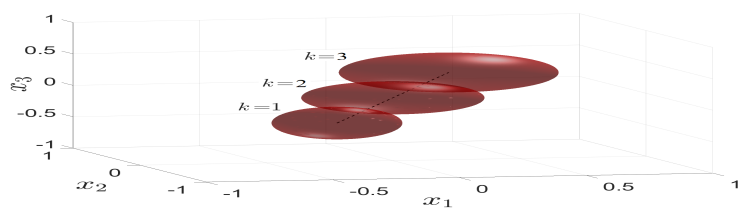

Fig. 15: Outside of the red shapes represent inner approximations of $\mathcal{C}_{r}^{\Delta=0.1}$ for time steps $k=1,2,3$.

Remark 3: In the provided examples, we addressed risk bounded motion planning problems in 2D and 3D environments. Recently, "Sparse Bounded degree SOS (SBSOS)" [26] and "Diagonally-Dominant SOS (DSOS)" ([27], [28]) techniques are introduced to solve large-scale SOS optimization. We can use these techniques to solve SOS optimizations of this paper for higher-dimensional problems (e.g., risk bounded motion planning in joint space).

\section{CONCLUSION}

In this paper, we addressed a novel approach to deal with perception uncertainties. For this purpose, we introduced risk contours map that shows the risk information of different regions in uncertain environment. To build such map, we used the notion of $\Delta$ risk contour that represents a set of points whose risk is less than or equal to the given risk level $\Delta$. We provided systematic numerical procedure to obtain outer and inner approximations of $\Delta$-risk contours in presence of nonconvexities and both bounded and unbounded probabilistic uncertainties. We leveraged recent results on chance constrained optimization and sum of squares optimizations. Several numerical examples are presented 
to illustrate the performance of the proposed approach.

\section{AppendiX A}

Here, we provide the sketch of the proof based on the recent results on chance constrained optimization (for more information see [15], [16], [20]). We assume that sets $\mathcal{K}_{o b s}$ and $\mathcal{K}_{\text {safe }}$ are compact. To satisfy this assumption, one can add $M-\|x\|_{2}^{2}-\|\omega\|_{2}^{2} \geq 0$ for some $M>0$ to the description of the sets, ([17], [18], [15]). To obtain SOS optimization in (8) and find the outer approximation $\hat{\mathcal{C}}_{r}^{\Delta}$ defined in (10), we will take the following steps: 1) We first address the chance optimization problem of the form "max $x_{x \in \chi} \operatorname{Prob}\left\{x \in \chi_{\text {safe }}(\omega)\right\}$ " and leverage the theory of moments to obtain the convex relaxation in the moment space, 2) We then use the duality theorem to find the dual optimization in the form of SOS optimization, 3) Finally, we leverage the SOS optimization of step (2) to obtain the SOS optimization in (8) and (10).

Step 1): Consider the chance optimization problem of the form $\max _{x \in \chi} \operatorname{Prob}\left\{x \in \chi_{\text {safe }}(\omega)\right\}$. To solve this nonconvex chance optimization, we want to find a convex relaxation. For this purpose, we take the following steps: 1-i) We find an equivalent optimization in space of distributions. For this, we assign an unknown probability distribution $\mu_{x}(x)$ defined on $\chi$, i.e., $\operatorname{supp}\left(\mu_{x}\right) \subset \chi$, to $x$. We then translate the chance optimization problem in terms of probability distributions $\mu_{\omega}(\omega), \mu_{x}(x)$, and a slack distribution $\mu(x, \omega)$ defined on $\left\{(x, \omega) \in \mathcal{K}_{\text {safe }}\right\}$. This results in the following "infinite dimensional" linear program in distribution space (Problem 3.2 and Theorem 3.1 in [15]): $\max _{\mu_{x}, \mu} \int d \mu$, s.t. $\mu \leq \mu_{x} \times \mu_{\omega}, \int d \mu_{x}=$ $1, \operatorname{supp}(\mu) \subset\left\{(x, \omega) \in \mathcal{K}_{\text {safe }}\right\}, \operatorname{supp}\left(\mu_{x}\right) \subset \chi$.

1-ii) Next, to find a tractable convex optimization, instead of looking for distributions $\mu_{x}$ and $\mu$ in step (1-i), we look for their moment sequences. For this purpose, we translate the cost function and constraints of obtained optimization in step (1-i) in terms of the moment sequences of the distributions. For this, we need the following lemma that shows the relation between a distribution and its moments ([17], [18], [15]). "Lemma: The sequence $\mathbf{y}=\left\{y_{\alpha}\right\}$ is a moment sequence of a distribution $\mu$ defined on the compact set $\{x: \mathcal{P}(x) \geq 0\}$, if and only if the following linear matrix inequalities hold $M_{d}(\mathbf{y}) \succcurlyeq 0, M_{d}(\mathbf{y} ; \mathcal{P}) \succcurlyeq 0, d \in \mathbb{N}$.", where $M_{d}(\mathbf{y})$ is the "moment matrix" in terms of the moments up to order $d$ and $M_{d}(\mathbf{y} ; \mathcal{P})$ is the "localizing matrix" in terms of the coefficients of the polynomial $\mathcal{P}$ and moments up to order $d$. Note that this lemma is valid for distributions that satisfy Assumption 2. This lemma, results in the following convex optimization in the moment space (Problem 3.6 and Lemma 3.2 in [15]): $\sup _{\mathbf{y}, \mathbf{y}_{\mathbf{x}}}(\mathbf{y})_{\mathbf{0}}$, s.t. $M_{d}(\mathbf{y}) \succcurlyeq 0, M_{d}(\mathbf{y} ; \mathcal{P}) \succcurlyeq 0, M_{d}\left(\mathbf{y}_{\mathbf{x}}\right) \succcurlyeq$ $0,\left\|\mathbf{y}_{\mathbf{x}}\right\| \leq 1,\left(\mathbf{y}_{\mathbf{x}}\right)_{\mathbf{0}}=1, M_{d}\left(\mathbf{y}_{\omega} \times \mathbf{y}_{\mathbf{x}}-\mathbf{y}\right) \succcurlyeq 0$, where $\mathbf{y}_{\mathbf{x}}$, $\mathbf{y}_{\omega}$, and $\mathbf{y}$ are the sequence of moments of distributions $\mu_{x}(x), \mu_{\omega}(\omega)$, and $\mu(x, \omega)$, respectively. Also, $(\mathbf{y})_{0}$ is the first element of the moment sequence $\mathbf{y}$ and $\mathcal{P}$ is the polynomial describing the uncertain safe region. Optimal value of the convex optimization in moment space is an upper bound of the optimal value of the original chance optimization of step (1) and monotonically converges as $d$ increase, ([15], [16]).

Step 2: Now, we apply the standard results on the duality of convex optimizations to the optimization of step (1-ii) and obtain the dual convex optimization in polynomial space. Given a standard form of a convex optimization involving linear matrix inequities as: $\min \langle C, X\rangle$, s.t. $\left\langle A_{i}, X\right\rangle=b_{i}, i=$ $1, \ldots, m, X \succcurlyeq 0$, where, $A_{i}, C \in \mathbb{R}^{n} \times \mathbb{R}^{n}$, vector $b \in \mathbb{R}^{m}$, and $X \in \mathbb{R}^{n} \times \mathbb{R}^{n},\langle C, X\rangle=\operatorname{trace}(C X)$, the dual optimization reads as ([17], [18], [19], [16]): $\max b^{T} y$, s.t. $C-\sum_{i=1}^{m} A_{i} y_{i} \succcurlyeq 0$. This will results in a SOS optimization in space of polynomials as follows [16]: $\min _{\hat{\mathcal{P}} \in \mathbb{R}_{d}[x, \omega]} \int_{\Omega} \hat{\mathcal{P}}(x, \omega) \mu_{\omega}(\omega) d \omega$, s.t. $\hat{\mathcal{P}}(x, \omega)-1 \geq$ 0 on $\left\{\forall(x, \omega) \in \mathcal{K}_{\text {safe }}\right\}, \hat{\mathcal{P}}(x, \omega) \geq 0$.

Step 3: In the chance optimization problem of step (1), we are looking for the point $x \in \chi$ that has the minimum risk of collision. Now, we leverage the obtained results in steps (1) and (2) to look for a set of all points whose risk is less than or equal to $\Delta$, i.e., $\mathcal{C}_{r}^{\Delta}$. For this, we extend the SOS optimization of step (2) that results in the following optimization [20]: $\min _{\hat{\mathcal{P}} \in \mathbb{R}_{d}[x, \omega]} \int_{\chi \times \omega} \hat{\mathcal{P}}(x, \omega) \mu_{\omega}(\omega) d \omega d x$, s.t. $\quad \hat{\mathcal{P}}(x, \omega)-$ $1 \geq 0$ on $\left\{\forall(x, \omega) \in \mathcal{K}_{\text {safe }}\right\}, \hat{\mathcal{P}}(x, \omega) \geq 0$. Not that the only difference between this SOS optimization and SOS optimization of step (2) is the cost function. This is the result of replacing $\mu_{x}$ in the optimization of step (1-i) with $\mu_{x}=d x$ (Eq. 12 and 13 and Theorem 3.1 in [20]). Then, $\mathcal{P}_{\text {outer }}(x)=\int_{\Omega} \hat{\mathcal{P}}(x, \omega) \mu_{\omega}(\omega) d \omega$ is an upper bound on the $\operatorname{Prob}\left(x \in \chi_{\text {safe }}\right)$ and converges as $d$ increases. Hence, the set $\left\{x \in \chi: \mathcal{P}_{\text {outer }}(x) \geq 1-\Delta\right\}$ forms a outer approximation of the $\mathcal{C}_{r}^{\Delta}$ and converges as $d$ increases, [20].

\section{Appendix B}

In Theorem 1, we obtain outer approximations of the set $\left\{x \in \chi: \operatorname{Prob}\left(x \in \chi_{\text {safe }}(\omega)\right) \geq 1-\Delta\right\}$. We can take the same steps to obtain outer approximations of the set $\left\{x \in \chi: \operatorname{Prob}\left(x \in \chi_{o b s}(\omega)\right)>\Delta\right\}$, (for more information on chance constrained optimization of complement of a set see [20]).

\section{REFERENCES}

[1] L. Blackmore, H. Li, B. C. Williams,"A probabilistic approach to optimal robust path planning with obstacles", American Control Conference (ACC), Minneapolis, 2006. 
[2] L. Blackmore, M. Ono, "Convex chance constrained predictive control without sampling", AIAA Guidance, Navigation, and Control Conference, Chicago, 2009.

[3] M. Ono, B. C. Williams, "Iterative risk allocation: A new approach to robust model predictive control with a joint chance constraint", IEEE Conference on Decision and Control (CDC), Cancun, Mexico, 2008.

[4] A. Jasour, A. Hofmann, and B.C. Williams, "Moment-Sum-OfSquares Approach For Fast Risk Estimation In Uncertain Environments", IEEE Conference on Decision and Control (CDC), Florida, USA, 2018

[5] W. Liu, M. H. Ang, "Incremental sampling-based algorithm for risk-aware planning under motion uncertainty", IEEE International Conference on Robotics and Automation (ICRA), Hong Kong, 2014.

[6] L. Janson, E. Schmerling, M. Pavone, "Monte Carlo motion planning for robot trajectory optimization under uncertainty", Springer Proceedings in Advance Robotics Book Series 3, Robotics Research, vol. 2, pp. 343-361, 2018.

[7] E. Schmerling, M. Pavone, 'Evaluating trajectory collision probability through adaptive importance sampling for safe motion planning” Robotics: Science and Systems (RSS), MIT, 2017.

[8] A. Pierson, W. Schwarting, S. Karaman, and D. Rus, Navigating Congested Environments with Risk Level Sets, , IEEE International Conference on Robotics and Automation (ICRA), Australia 2018.

[9] A. A. Ahmadi, G. Hall, A. Makadia, V. Sindhwani, "Geometry of 3D environments and sum of squares polynomials", Robotics: Science and Systems (RSS), MIT, 2017.

[10] A. Majumdar, A. A. Ahmadi, and R. Tedrake, "Control Design Along Trajectories via Sum of Squares Optimization", International Conference on Robotics and Automation (ICRA), MN USA, 2012.

[11] S. Shen and R. Tedrake,"Compositional verification of large-scale nonlinear systems via sums-of-squares optimization", American Control Conference (ACC), Milwaukee, USA, 2018.

[12] M. Posa, T. Koolen, and R. Tedrake, "Balancing and step recovery capturability via sums-of-squares optimization”, Robotics: Science and Systems, MIT, 2017.

[13] R. Tedrake, I R. Manchester, M. M. Tobenkin, and J. W. Roberts, "LQR-Trees: Feedback motion planning via sums of squares verification", International Journal of Robotics Research, 29:10381052, July, 2010.

[14] A. Majumdar, Russ Tedrake, "Funnel libraries for real-time robust feedback motion planning", International Journal of Robotics Research, 36(8):947 - 982, 2017

[15] A. Jasour, N. S. Aybat, C. Lagoa "Semidefinite programming for chance constrained optimization over semialgebraic sets, SIAM Journal on Optimization, 25(3), 1411-1440, 2015.

[16] A. Jasour, "Convex Approximation of Chance Constrained Problems: Application in Systems and Control", School of Electrical Engineering and Computer Science, The Pennsylvania State University, 2017.

[17] J. B. Lasserre, "Global optimization with polynomials and the problem of moments", SIAM Journal on Optimization, vol. 11, pp. 796-817, 2011.

[18] M. Laurent, "Sums of squares, moment matrices and optimization over polynomials", In: Putinar M., Sullivant S. (eds) Emerging Applications of Algebraic Geometry. The IMA Volumes in Mathematics and its Applications, vol. 149, Springer, New York, 2009.

[19] P. A. Parrilo, "Semidefinite programming relaxations for semialgebraic problems", Mathematical Programming, vol. 96, pp. 293320, 2003.

[20] J. B. Lasserre, "Representation of chance-constraints with strong asymptotic guarantees", IEEE Control Systems Letters, 1 (1), pp.50-55, 2017.

[21] S. Karaman, E. Frazzoli, "Sampling-based algorithms for optimal motion planning", The International Journal of Robotics Research, 30(7), 846-894, 2011.

[22] L., Johan, "Pre- and post-processing sum-of-squares programs in practice", IEEE Transactions on Automatic Control, vol. 54, 1007-1011, 2009.

[23] F. Dabbene, D. Henrion, "Set approximation via minimumvolume polynomial sublevel sets", European Control Conference (ECC), Switzerland, 2013.

[24] Sh. Dong, B. C. Williams," Learning and recognition of hybrid manipulation motions in variable environments using probabilistic flow tubes", International Journal of Social Robotics, 4(4):357368, 2012.

[25] R. L. H. Deits. R. Tedrake, "Computing large convex regions of obstacle-free space through semidefinite programming", In Proceedings of the Eleventh International Workshop on the Algorithmic Foundations of Robotics (WAFR 2014), Istanbul, 2014.

[26] T. Weisser, J.B. Lasserre, and KC. Toh, "Sparse-BSOS: a bounded degree SOS hierarchy for large scale polynomial optimization with sparsity", Mathematical Programming Computation ,Volume 10, Issue 1, pp 1-32,2018.

[27] A. Majumdar, A. A. Ahmadi, and R. Tedrake "Control and verification of high-dimensional systems with DSOS and SDSOS programming", IEEE conference on decision and control (CDC), Los Angeles, CA, 2014.

[28] A. A. Ahmadi, A. Majumdar, "DSOS and SDSOS Optimization: More Tractable Alternatives to Sum of Squares and Semidefinite Optimization", To appear in SIAM Journal on Applied Algebraic Geometry, arXiv:1706.02586, 2018. 